\title{
Biomarkers of drug-induced liver injury
}

This article was published in the following Dove Press journal:

Current Biomarker Findings

II January 2013

Number of times this article has been viewed

\author{
Yong Zhou \\ Shizhen Qin \\ Kai Wang \\ Institute for Systems Biology, Seattle, \\ WA, USA
}

Correspondence: Kai Wang Institute for Systems Biology 40I Terry Avenue North Seattle, Washington 98109, USA $\mathrm{Tel}+\mid 206732$ |336

Fax + I 206732 I299

Email kwang@systemsbiology.org
Abstract: The liver plays a central role in metabolizing xenobiotics; therefore, it is highly susceptible to toxicity from these chemicals. Certain drugs, when taken in overdose and sometimes even when used within therapeutic range, may cause injury to the organ. Druginduced liver injury is now not only a leading cause of acute liver failure in the US, but is also a leading reason for discontinuation of drugs in development and for regulatory actions against previously approved drugs. The current clinical biomarkers to detect and monitor drug-induced liver injury are inadequate in terms of sensitivity and/or specificity, prompting the need for more informative biomarkers. The development of high throughput proteomics, genomics, and metabolomics technologies has the potential to fulfill such demand. The discipline of systems toxicology may add to our understanding of perturbed xenobiotic networks, which may lead to network-specific surrogate markers and therapeutic means to stop or reverse xenobiotic-induced liver injury.

Keywords: hepatotoxicity, idiosyncratic, metabolomics, genomics, proteomics, microRNA, systems toxicology

\section{Introduction}

Adverse effects associated with medicine used in clinics are a serious problem for patients and health care providers. ${ }^{1}$ It has been estimated that about $10 \%$ of drugs are associated with severe, undesirable side effects. ${ }^{2,3}$ However, the number is probably significantly underestimated given that drug-induced adverse effects are difficult to detect due to pre-existing medical conditions, multiple drug usage, and lack of diagnostic standards. ${ }^{1}$ Among all the major organs in the body, most drug-induced adverse effects are associated with the liver due to its active role in metabolizing xenobiotics. While the overall incidence of drug-induced liver injury (DILI) in the general population is largely unknown, the incidence of DILI attributed to an individual drug is estimated to be between one in 10,000 and one in 1,000,000 patient years for most medicines used in the clinic. ${ }^{4,5}$ Although the precise incident rate for DILI is difficult to establish, it has become the leading cause of drug development failure and post-approval usage restrictions or withdrawals. ${ }^{1,6}$ DILI is now the leading cause of acute liver failure, exceeding all other causes combined in Europe and North America. ${ }^{1,7,8}$ To address this challenging and often controversial issue, significant efforts have been focused on the identification of informative and predictive biomarker signatures for DILI through various globalomics-based molecular profiling approaches. ${ }^{9-11}$ In addition, multicenter research networks, such as the Drug-Induced Liver Injury Network, have begun to collect biological samples and develop approaches submit your manuscript | www.dovepress.com

Dovepress

http://dx.doi.org// 0.2/47/CBF.S27900
Current Biomarker Findings 2013:3 I-9

(C) 2013 Zhou et al, publisher and licensee Dove Medical Press Ltd. This is an Open Access article which permits unrestricted noncommercial use, provided the original work is properly cited. 
for the diagnosis, evaluation, and reporting of patients with suspected liver injury caused by the use of therapeutics and/ or herbal products. ${ }^{9}, 12$

\section{Pathogenic mechanism of DILI}

Even though the etiology of DILI is complicated and largely unknown, current knowledge can generally group the mechanisms into two major categories, ie, direct toxicity and indirect toxicity (Figure 1). Direct toxicity includes injuries caused directly by the xenobiotic or its metabolites, such as the toxic metabolite $N$-acetyl- $p$-benzoquinoneimine derived from acetaminophen. ${ }^{13}$ In conditions with high doses of acetaminophen exposure or low cellular glutathione concentration, $N$-acetyl- $p$-benzoquinoneimine binds to proteins and other macromolecules, interrupting their normal cellular activities, and leading to apoptosis of hepatocytes and centrilobular necrosis in the liver. ${ }^{14}$ Fortunately, most drug candidates and metabolites that induce significant direct toxicity can be screened out during the drug development process and seldom reach the market. However, obtaining a full safety profile for drugs is simply not possible due to cost and factors such as rare polymorphisms in drugmetabolizing enzymes and host immune response genes, as well as environmental factors. Certain drugs affecting a small number of patients can escape various safety screening steps in the drug development process and reach the market. ${ }^{2,15}$ For example, perhexiline [2-(2,2-dicyclohexylethyl)piperidine], an effective antianginal drug, when used in high doses, causes severe neurotoxicity and hepatotoxicity. ${ }^{16}$ However, at

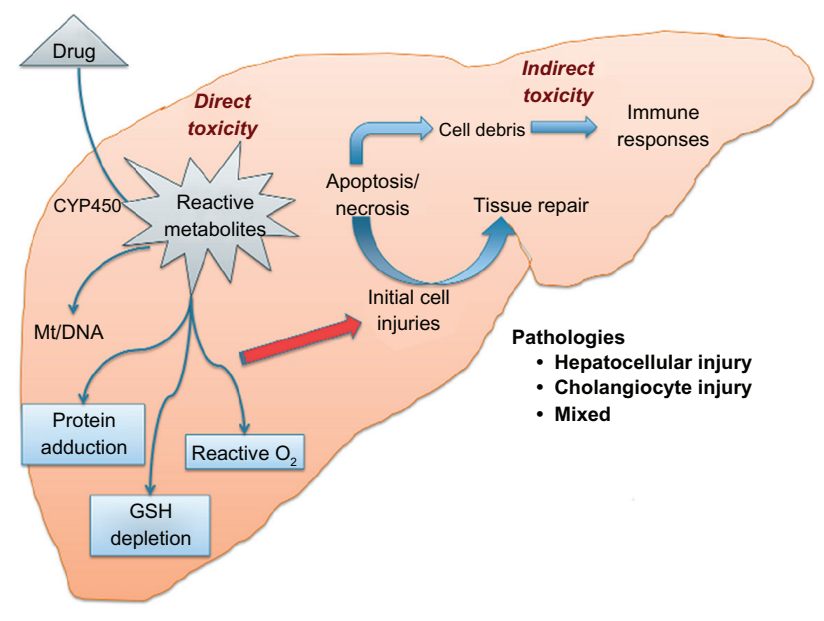

Figure I Schematic diagram of key events involved in drug-induced liver injury. Notes: Processes from drug metabolism, activation of innate immune responses, and direct and indirect hepatocyte damage are all involved in the initiation and progression of tissue injuries.

Abbreviations: CYP450, cytochrome P450 isozymes; GSH, glutathione; Mt, mitochondria. a standard dosage, certain individuals with mutations in cytochrome P450 2D6 (CYP2D6) isozyme, the key enzyme for metabolizing perhexiline, develop adverse effects due to their inability to process and excrete the drug at a normal rate. ${ }^{17}$ As a result, the drug was withdrawn from most countries in the late $1980 \mathrm{~s}$.

Indirect toxicity is a more complicated and less understood process. It usually involves an inflammatory process, including the activation of innate and/or adaptive immune responses. ${ }^{18}$ Sometimes additional events such as viral and/or bacterial infections and other drug usage are required to induce indirect toxicity. ${ }^{8}$ Minor hepatocellular dysfunction and cell death caused by therapeutic drugs or other factors may trigger the activation of cells involved in the innate immune system, such as Kupffer cells (resident macrophages of the liver) and natural killer cells. ${ }^{19}$ These cells may then exacerbate an initial minor injury by activating the adaptive immune system and producing proinflammatory mediators to recruit additional inflammatory cells to the liver. ${ }^{20}$ The key evidence that drugs activate the adaptive immune response is the ability to detect antibodies against drugs or their metabolites. For example, autoantibodies have been reported for halothane, dihydralazine, diclofenac, carbamazepine, tienilic acid, to name just a few. ${ }^{21}$ However, the link between activation of the adaptive immune system and DILI is still unclear. Despite the ability to segregate direct and indirect toxicity, the underlying mechanism for both types of DILI is not only complicated, but also difficult to study with existing technologies.

Based on the incident pattern, there are two different types of DILI, predictable and idiosyncratic. ${ }^{22}$ Predictable toxicity usually shows a dose dependency with a short latency. Most predictable DILI is induced by drugs associated with direct toxicity. ${ }^{23}$ Acetaminophen is likely the most studied hepatotoxic drug, and gives a predictable toxicity in a dose-dependent fashion. ${ }^{14,19}$ Besides acetaminophen, there are a number of other drugs or supplements which are also known to cause predictable DILI. For example, amiodarone $^{24}$ (an antiarrhythmic drug), isoniazid ${ }^{25}$ (the first-line drug in treating tuberculosis), and kava kava ${ }^{26}$ (an herbal supplement) are all known to show predictable dosedependent hepatotoxicity.

However, most instances of liver injury caused by drugs are unpredictable, and are termed idiosyncratic DILI. ${ }^{14,27,28}$ Unlike predictable adverse reactions, idiosyncratic reactions show no relationship with dose and occur with different latency, from one week to more than a year after the drug treatment. ${ }^{2}$ Idiosyncratic adverse reactions can be related 
to interactions between the drug and host or environmental factors. In addition, how the host responds to the initial insults from drugs or their metabolites, including the ability to adapt, repair, and temper innate and adaptive immunity, might be as important as the factors involved in the initiation of liver injury.

\section{Types of pathology}

Even though all conceivable injuries induced by drugs have been seen in the liver, the injuries can generally be grouped into four major patterns, ie, the hepatitis, cholestatic, mixed, and other patterns based on the pathology (Figure 1). ${ }^{2}$ The hepatitis pattern is usually associated with hepatocellular injury. Patients with hepatitis display a wide spectrum of clinical manifestations from asymptomatic, fatigue, and pain to acute liver failure. ${ }^{29}$ Individuals usually show higher levels of serum aminotransferase activities. However, biochemical and clinical parameters often underestimate the degree of injury. Drugs such as acetaminophen, amiodarone, and isoniazid are known to cause hepatitis pattern DILI. ${ }^{30}$

The cholestatic pattern is usually caused by cholangiocyte injury or inhibition of bilirubin or bile salt transport. ${ }^{31}$ Patients usually develop jaundice with elevation of blood alkaline phosphatase levels. ${ }^{32}$ This type of injury is usually less serious than the hepatitis pattern and typically does not develop into chronic liver disease. ${ }^{4}$ Drugs including amoxicillin, ${ }^{33}$ chlorpromazine, ${ }^{34}$ erythromycin,${ }^{35}$ and estrogens ${ }^{36}$ are known to cause cholestatic DILI.

Certain drugs such as phenytoin, ${ }^{37}$ phenobarbital, ${ }^{38}$ and verapamil ${ }^{39}$ are known to cause a mixed type injury, a combination of hepatitis and cholestatic patterns. Patients usually have higher levels of both aminotransferases and alkaline phosphatase activities. This type of injury has a lower mortality rate compared with that of individuals with either hepatitis or cholestatic patterns. ${ }^{4}$ In addition to the three major patterns, certain drugs also induce other liver pathologies, including granulomas, fibrosis, neoplasms, steatohepatitis, and vascular lesions. ${ }^{40}$

\section{Genetic markers used in detecting DILI}

Even though it does not account for all the risk factors, DILI is strongly affected by genetic predisposition, and especially by the polymorphisms associated with type I and II drug metabolizing enzymes. ${ }^{41,42}$ Various genetic polymorphisms in drug metabolizing enzymes and immunological mediators such as human leukocyte antigen (HLA) molecules and cytokines have been shown to correlate well with the patients' susceptibility to DILI. ${ }^{43,44}$ A comprehensive list of the candidate genes can be found in recent reviews. ${ }^{45,46}$ The polymorphisms of these genes may be able to serve as markers to predict susceptibility of exposed individuals to DILI.

Advanced technologies such as genome-wide association studies (GWAS) and highly parallelized nextgeneration sequencing (NextGen) are now providing new methods to explore the association between genetic predisposition and DILI. For example, a GWAS study revealed that the HLA-B*5701 genotype is a major risk factor and ST6GAL1 is a possible cofactor of an individual's vulnerability to flucloxacillin-induced DILI. ${ }^{47}$ Whether the HLA-B*5701 genotype could serve as a new DILI biomarker for flucloxacillin warrants further investigation. At present, the costs of GWAS or NextGen sequencing is still too high to be used in routine clinic screening. In addition, the predictive value for these genetic biomarkers to DILI is yet to be determined. ${ }^{3,11}$ Thus, at the present time, testing patients for the presence of susceptible genetic polymorphisms before prescribing a drug with potential DILI has very low clinical value. ${ }^{48,49}$ However, as more and more information is collected, it will be feasible to have a comprehensive pharmacogenetic test prior to prescribing certain drugs to determine an individual's susceptibility to DILI, just like the association between CYP2C9 polymorphism and warfarin usage. ${ }^{50,51}$ The US Food and Drug Administration (FDA) has started a pilot process to explore the use of genomic biomarkers in FDA-approved drug labels. For example, $\mathrm{N}$-acetyltransferase variants (associated with slow and fast acetylators of antituberculosis drugs $)^{52}$ and the HLA-B*5701 allele (associated with the anti-human immunodeficiency virus drug abacavir), ${ }^{47}$ as potential genomic susceptibility markers for DILI.

\section{Blood biomarkers used in detecting DILI}

In addition to genetic markers, histological examination and imaging, the most common tests used in the clinic are based on the concentration changes of specific molecules in different body fluids, especially blood. Blood contains an array of biomolecules from small molecules to proteins. Many of the molecules in the circulation are the results of either cell lysis or metabolites to be excreted from the body. Therefore, the levels of specific biomolecules in the circulation may be used to assess the health status of the body. ${ }^{53}$ Different types of blood DILI biomarkers including metabolites, proteins, and RNAs have been reported in human and animal models, and examples are listed in Table 1. 
Table I Examples of blood biomarkers for DILI and acute liver failure

\begin{tabular}{|c|c|c|c|c|c|}
\hline Name & Species & Body fluid & Drug compounds & Biomarker functions & Reference \\
\hline \multicolumn{6}{|l|}{ Metabolite } \\
\hline Ophthalmate & Rat & Serum & Acetaminophen & $\begin{array}{l}\text { Hepatic glutathione depletion } \\
\text { or oxidative stress }\end{array}$ & Soga et al ${ }^{54}$ \\
\hline 8-hydroxy-2'-deoxyguanosine & Human & Urine & Valproic acid & & Lee et $\mathrm{al}^{55}$ \\
\hline Octanoylcarnitine & Human & Urine & Valproic acid & & Lee et $\mathrm{al}^{55}$ \\
\hline Galactose & Rat & Blood & $\begin{array}{l}\mathrm{CCl}_{4}, \mathrm{INH} \text { and } \mathrm{INH} \\
+\mathrm{BNPP}\end{array}$ & Liver functions & Young et $\mathrm{al}^{56}$ \\
\hline \multicolumn{6}{|l|}{ Protein } \\
\hline FL-KI8 (M65)/cKI8 (M30) & Human & Serum & Acetaminophen & $\begin{array}{l}\text { Hepatocytic necrosis/ } \\
\text { apoptosis/outcome }\end{array}$ & $\begin{array}{l}\text { Antoine et al }{ }^{63} \\
\text { Rutherford et al }\left.\right|^{64}\end{array}$ \\
\hline HMGBI (total/acetylated) & Human & Serum & Acetaminophen & $\begin{array}{l}\text { Hepatocytic necrosis/immune } \\
\text { cell activation }\end{array}$ & Antoine et $\mathrm{al}^{63}$ \\
\hline GDH & Human & Plasma & Acetaminophen & Mitochondrial dysfunction & McGill et al ${ }^{62}$ \\
\hline Caspase activation & Human & Plasma & Acute liver failure & Liver regeneration after ALF & Volkmann et $\mathrm{al}^{\mid 61}$ \\
\hline \multicolumn{6}{|l|}{ DNA/RNA } \\
\hline DNA (mt/nuclear DNA fragments) & Human & Plasma & Acetaminophen & $\begin{array}{l}\text { Mt dysfunction/nuclear DNA } \\
\text { damage }\end{array}$ & McGill et $\mathrm{a}^{62}$ \\
\hline RNA (Alb, Fga, and Hp) & Rat & Blood & Acetaminophen/D-gal & & Miyamoto et al ${ }^{66}$ \\
\hline MicroRNA miR-I22, miR-192 & Mouse & Plasma & Acetaminophen & & Wang et $\mathrm{al}^{68}$ \\
\hline MicroRNA miR-I22 & Mouse & Plasma & D-gal/alcohol & & Zhang et $\mathrm{al}^{71}$ \\
\hline MicroRNA miR-I 22 & Human & Plasma & Chronic hepatitis B & & Zhang et $\mathrm{al}^{71}$ \\
\hline
\end{tabular}

Abbreviations: Alb, albumin; ALF, acute liver failure; BNPP, bis-p-nitrophenyl phosphate; $\mathrm{CCl}_{4}$, carbon tetrachloride; cK I8, caspase-cleaved fragment of keratin-18; DILI, drug-induced liver injury; Fga, fibrinogen alpha chain; FL-KI8, full length keratin- I8; D-gal, D-(+)-galactosamine; GDH, glutamate dehydrogenase; HMGB-I, high mobility group box-I; HP, haptoglobin; INH, isoniazid; mt, mitochondrial.

\section{Metabolite markers}

Metabolomics studies aim to reveal the profiles of smallmolecule metabolites produced by cellular processes of an organ or the whole organism. In animal models, metabolomics investigations have identified a growing number of DILI biomarkers from different types of body fluids, such serum and urine. For example, using a rat acetaminophen DILI model, serum ophthalmate was found to be a sensitive biomarker to reflect hepatic glutathione depletion or oxidative stress. ${ }^{54}$ The concentrations of 8-hydroxy-2'-deoxyguanosine and octanoylcarnitine in urine were also identified as indicators for valproic acidinduced DILI. ${ }^{55}$ The change in blood galactose levels has also been shown to be a better liver function indicator than the blood aminotransferase activities in animal models. ${ }^{56}$ The noninvasive nature of metabolomics markers in different types of body fluid samples makes them attractive; however, as with any biomarker identified, they require extensive validation with a large number of biological samples. ${ }^{57}$

\section{Protein markers}

The most widely adopted DILI blood biomarkers are protein in nature, especially enzymes that are highly enriched in the liver, including aminotransferases, glutamate dehydrogenase, lactate dehydrogenase, and alkaline phosphatase. It is believed that when liver injury occurs, these abundant enzymes in hepatocytes leak into the blood stream. ${ }^{58}$ The changes in the activities or concentrations of these enzymes in blood should then reflect the degree of injury to the liver. The most commonly used aminotransferases for detecting DILI are glutamic pyruvate transaminase (GPT, also known as alanine aminotransferase) and glutamic-oxaloacetic transaminase, (GOT, also known as aspartate aminotransferase). ${ }^{3}$ Due to its molecular size, the half-life of GPT in serum is about $40-60$ hours. ${ }^{58}$ An elevation of serum GPT activity is therefore an indication of damage that has occurred within the past $40-60$ hours. Although it is generally believed that most of the enzyme activity tests have sufficient sensitivity, their specificity to DILI is often questionable. Extrahepatic expression of these liver-enriched enzymes is the main drawback for these markers. Therefore, injuries in other tissues may also cause changes in levels of these enzyme in blood..$^{59}$ The lack of tests to distinguish different GOT and GPT isoenzyme activities further limit its usage. Despite these problems, elevation of serum aminotransferase levels/activities are still the most commonly used tests by pharmaceutical companies and regulatory agencies as indicators for DILI. ${ }^{60}$ 
In addition to liver-enriched proteins and enzymes, many cytokines such as tumor necrosis factor alpha, interleukin (IL) $1 \beta$ and interleukin- 6 have also been used as indicators for DILI, because inflammation is one of the key events in the initiation and progression of DILI. ${ }^{46}$ Recent epidemiological studies have also reported levels of several circulating proteins and their ratios as indicators/predictors for hepatocyte damage and repair. ${ }^{61,62}$ These proteins include full length cytokeratin-18 (K18), caspase-cleaved fragment of K18 (M30, indicating apoptosis and necrosis), high mobility group box-1 (HMGB1, indicating necrosis) and hyperacetylated HMGB1 (indicating apoptosis and immune cell activation). ${ }^{63}$ Combining the M30 level with clinical variables, including serum $\mathrm{pH}$, body mass index, levels of creatinine, bilirubin, phosphorus, arterial ammonia, and lactate, gave a good association with the outcomes in patients with acute liver failure. ${ }^{64}$

Zimmerman proposed the most widely adapted comprehensive test to detect DILI. The test includes the activities/ concentrations of four different molecules in serum, GPT, GOT, alkaline phosphatase, and total serum bilirubin. These four biomarkers, along with an elevation of aminotransferases above three times the upper limit of normal and total serum bilirubin more than twice the upper limit of normal, have been known as Hy's Law which is more effective in detecting serious liver injuries than elevation of aminotransferases alone. ${ }^{65}$

\section{RNA markers}

Besides protein markers, several blood messenger RNA-based markers have also been shown to have good correlations with DILI. In rat DILI models, the levels of several "liver-specific" transcripts including albumin, fibrinogen, and haptoglobin genes in blood show significant elevations, along with blood aminotransferase activity after exposing rats to either acetaminophen or D-(+)-galactosamine. ${ }^{66}$ The concentrations of these mRNAs in blood were not affected if the animals were treated with bupivacaine, a chemical that causes muscle injury. However, in bupivacaine-treated rats, the levels of aminotransferase activity increased significantly in blood. ${ }^{67}$ This demonstrated the specificity of using mRNA-based biomarkers over traditional aminotransferase (protein)-based biomarkers to detect DILI.

Besides mRNA, a number of microRNAs in blood have also been reported as markers for DILI. ${ }^{68}$ MicroRNAs are small, single-stranded noncoding regulatory RNA molecules with an average length of about 20 nucleotides. They play a key role in modulating transcript and protein levels in cells. ${ }^{69}$ Since their discovery in the early 1990s, studies have shown that some of these important regulatory RNA molecules exist stably in various types of body fluid including blood and urine. The levels of some extracellular microRNAs show good correlations with various pathological conditions, such as cancers of various origins. The concentrations of two liver-enriched microRNAs in plasma, miR-122, and miR-192, were first found to have a good correlation with acetaminophen overdose induced-liver injury in animal models. ${ }^{68,70}$ The plasma levels of these two microRNAs show better sensitivity and specificity than blood GPT levels. The circulating miR-122 levels also reflected DILI induced by $\mathrm{D}$-(+)-galactosamine. ${ }^{71} \mathrm{~A}$ more recent study showed that circulating miR-122 levels, but not serum GPT and GOT, could effectively differentiate liver injury from extrahepatic (ie, heart or muscle) injury. ${ }^{71}$ Based on the findings from these studies, the levels of specific circulating microRNAs in blood may perform better than the standard aminotransferase-based DILI biomarkers.

It is important to note that there is currently no perfect biomarker for DILI. Despite this deficiency, the combination of serum GPT, GOT, alkaline phosphatase, and total bilirubin levels continues to be the standard test for DILI, especially when measured consistently over the period of drug treatment. ${ }^{72}$ In the past decade, some progress has been made in developing better DILI biomarkers, such as attempts to develop isoenzyme-specific measurements of serum GPT levels and liver-enriched miRNA and mRNA levels in the circulation. ${ }^{73}$ Drug-specific biomarkers, such as blood acetaminophen-protein adducts, can also identify the etiology of DILI; however, this can only be applied for a limited number of drugs. ${ }^{74}$ It is worthwhile to point out that none of the above-mentioned new DILI biomarkers have been extensively validated.

\section{Approaches for new biomarker discovery}

While some DILI markers are effective in identifying potentially harmful drug candidates in the in vitro and in vivo models used in the drug development process, the current biomarkers lack the predictability, sensitivity, and specificity needed in the clinic. ${ }^{72}$ This is due to the diverse pathologies associated with drug-induced injuries and complex drug interactions in patients. More importantly, the current biomarkers are not able to pinpoint the type of injury, which is a vital necessity in clinic. ${ }^{14}$ Another major drawback is that the current DILI biomarkers are not suitable for detecting chronic liver injury. ${ }^{75}$ An example is chronic methotrexate-induced hepatotoxicity; there is no available 
marker to detect and monitor the injury effectively, which forces some clinicians to use routine serial liver biopsies as an alternative to reduce the occurrence of severe DILI associated with methotrexate. ${ }^{76}$ Because DILI is a serious problem in health care, there is an urgent need to have biomarkers that can provide information for early detection, sufficient sensitivity of the injury, types of DILI, and prognosis of the injuries. ${ }^{77}$ Markers that can serve as predictors of response to treatment in patients with DILI are also in need. ${ }^{49}$ The advancement of various high throughput global molecular profiling technologies for proteins and nucleic acids has the potential to lead to more informative biomarkers.

High performance mass spectrometry (MS)-based protein identification and measurement has emerged as a key technology used to identify and validate protein biomarkers. ${ }^{78}$ For discovery, the process can be divided into two major approaches, ie, labeled and label-free methods. Development of the isobaric tags for relative and absolute quantitation method has simplified global protein quantitation. ${ }^{79}$ The main advantage of this approach is to reduce operation-related sample-to-sample variations, because all samples are mixed and processed at the same time after being labeled with different isobaric tags. The label-free approach is mainly based on the unique mass/ charge $(\mathrm{m} / \mathrm{z})$ ratio of peptides to predict protein identity. This approach is computationally intense, and the accuracy of protein prediction depends on the completeness of the protein database. ${ }^{80}$ The drawback for MS-based biomarker discovery is the number of proteins (usually several hundred) that can be identified and quantified in each MS run. The recently developed selected reaction monitor (SRM) Atlas, combined with the "Swath" acquisition approach, significantly increases the number of proteins that can be detected and may have the capacity to systematically query any protein of interest in samples. ${ }^{81}$

Western blotting is the most commonly used method for validation. However, it can only be used on a limited number of samples and one protein marker at a time. It also depends on the availability of suitable antibodies. ${ }^{82}$ For a large number of samples, the MS-based selected reaction monitor approach is commonly used to quantify specific protein biomarkers accurately. ${ }^{83}$ This approach spikes in known concentrations of labeled heavy peptide(s) as a concentration standard, and uses mass spectrometer to detect and quantify the light (endogenous) and heavy (spiked in) peptides. The selected reaction monitor approach is also able to measure several proteins at the same time and is not limited to the availability of antibodies. ${ }^{84}$
Because most efforts at biomarker discovery aim to identify blood-based markers, the front-end sample processing plays an important role in the MS-based discovery approach. ${ }^{85}$ However, sample preparation can be a challenge due to the high complexity of blood samples. Protein concentrations in the circulation often span more than 10 orders of magnitude, and most of the proteins of interest in blood are at the lower end of the concentration spectrum. ${ }^{86}$ Enrichment processes, such as affinity column enrichment or depletion of highly abundant proteins, are a common practice for MS-based blood protein analysis. ${ }^{87}$ However, the need for significant front-end sample processing hinders the use of MS-based biomarker detection in the clinic. Once a protein biomarker is validated, the enzyme-linked immunosorbent assay is likely the most suitable method for routine clinic use, although developing proper reagents for an accurate and sensitive assay is nontrivial.

As with proteins, MS has evolved to be the most suitable platform to identify metabolomic markers. ${ }^{57}$ The small molecules in samples are usually fractionated via gas chromatography, high performance liquid chromatography, or capillary electrophoresis before injection into the MS for measurement. ${ }^{88}$ The human metabolome database (http://www.hmdb.ca) contains over 7000 small molecule metabolites derived from degradation of endogenous biomolecules, xenobiotics, and food components detected in the body. ${ }^{89}$ One of the biggest challenges in metabolomics is to pinpoint the origin of the metabolites. ${ }^{90}$ In the case of identifying biomarkers for xenobiotic-induced injuries, the xenobiotics themselves and their metabolites are useful markers to determine the drugs causing such effects. However, identifying the metabolites that reflect the type and severity of injury remains a challenge. ${ }^{57}$

Blood also contains a diverse range of RNA and DNA molecules. Several studies have already demonstrated the usefulness of using specific DNA or RNA sequences in the circulation, such as transcripts highly enriched in the liver, as markers to reflect DILI. ${ }^{11,67,91}$ The concentrations of these molecules are too low to conduct a global survey using traditional nucleic acid profiling approaches like microarray. Besides microRNA, due to its small repertoire, no systematic survey of the DNA or RNA spectrum in the circulation has been done. The recent development of next-generation sequencing requires much less starting material, which creates the possibility of conducting a comprehensive survey of the nucleic acid spectrum in blood to identify more informative nucleic acid-based biomarkers for xenobiotic-induced tissue injury. 
It is important to point out that despite our ability to generate a list biomarker candidates using systematic global profiling, a number of useful biomarkers such as K-8 and its fragment, and mitochondrial and nuclear DNA fragments, have been identified through traditional approaches. ${ }^{62,63}$

\section{Future prospects}

Despite the technical advances in profiling biomolecules, identification of biomarkers from plasma or serum still poses many challenges. These include the diversity and wide concentration distribution of biomolecules in the circulation, complex post-transcriptional and post-translational modifications, the low concentration of molecules of interest, and the origin of the biomolecules in circulation. However, the biggest challenge is the integration of such global survey results. Systems toxicology, a discipline derived from systems biology, aims to integrate the results of different global molecular measurements to discover perturbed networks, build testable models, identify surrogate markers to reflect the perturbed network, and find methods to modulate or reverse the affected networks. ${ }^{92}$ The integration of multidimensional measurement results, including genomic, transcriptomic, proteomic, and metabolomic measurements, are a challenge due to our lack of understanding of how biomolecules interact in cells. In addition, a better understanding of higher-level interactions, such as how cells interact with each other in tissues and how tissues communicate in an organism, is clearly desirable. The basis of system toxicology is to generate and use animal models to emulate the diversity of the human population, and to identify informative biomarkers and therapeutic strategies through the understanding of perturbed networks. Several studies using inbred mouse panels to explore the genetic contribution to the effects of xenobiotics are worth further investigation.

\section{Acknowledgments}

We thank Alton Etheridge for his critical comments. This work was supported by the University of Luxembourg program and research contracts from the Department of Defense (W911SR-07-C-0101, W81XWH-09-1-0107, and HDTRA 1-08-C-0023).

\section{Disclosure}

The authors report no conflicts of interest in this work.

\section{References}

1. Assis DN, Navarro VJ. Human drug hepatotoxicity: a contemporary clinical perspective. Expert Opin Drug Metab Toxicol. 2009;5(5):463-473.

2. Hussaini SH, Farrington EA. Idiosyncratic drug-induced liver injury: an overview. Expert Opin Drug Saf. 2007;6(6):673-684.
3. Shi Q, Hong H, Senior J, Tong W. Biomarkers for drug-induced liver injury. Expert Rev Gastroenterol Hepatol. 2010;4(2):225-234.

4. Rangnekar AS, Fontana RJ. An update on drug induced liver injury. Minerva Gastroenterol Dietol. 2011;57(2):213-229.

5. Sgro C, Clinard F, Ouazir K, et al. Incidence of drug-induced hepatic injuries: a French population-based study. Hepatology. 2002;36(2): 451-455.

6. Guengerich FP. Mechanisms of drug toxicity and relevance to pharmaceutical development. Drug Metab Pharmacokinet. 2011; 26(1):3-14.

7. Lee WM. Acute liver failure. Semin Respir Crit Care Med. 2012;33(1): 36-45.

8. Hadem J, Tacke F, Bruns T, et al. Etiologies and outcomes of acute liver failure in Germany. Clin Gastroenterol Hepatol. 2012;10(6):664-669, e662.

9. Bell LN, Vuppalanchi R, Watkins PB, et al. Serum proteomic profiling in patients with drug-induced liver injury. Aliment Pharmacol Ther. 2012;35(5):600-612.

10. Van Summeren A, Renes J, van Delft JH, Kleinjans JC, Mariman EC. Proteomics in the search for mechanisms and biomarkers of drug-induced hepatotoxicity. Toxicol In Vitro. 2012;26(3):373-385.

11. Mendrick DL. Transcriptional profiling to identify biomarkers of disease and drug response. Pharmacogenomics. 2011;12(2):235-249.

12. Agarwal VK, McHutchison JG, Hoofnagle JH. Important elements for the diagnosis of drug-induced liver injury. Clin Gastroenterol Hepatol. 2010;8(5):463-470.

13. Nelson SD. Molecular mechanisms of the hepatotoxicity caused by acetaminophen. Semin Liver Dis. 1990;10(4):267-278.

14. Tujios S, Fontana RJ. Mechanisms of drug-induced liver injury: from bedside to bench. Nat Rev Gastroenterol Hepatol. 2011;8(4):202-211.

15. McBurney RN, Hines WM, Von Tungeln LS, et al. The liver toxicity biomarker study: phase I design and preliminary results. Toxicol Pathol. 2009;37(1):52-64.

16. Long JP, Fitzgerald O, Maurer BJ. Hepatotoxicity following treatment with perhexiline maleate. Ir Med J. 1980;73(7):275-276.

17. Ashrafian H, Horowitz JD, Frenneaux MP. Perhexiline. Cardiovasc Drug Rev. 2007;25(1):76-97.

18. Uetrecht J. Immunoallergic drug-induced liver injury in humans. Semin Liver Dis. 2009;29(4):383-392.

19. Jaeschke H, McGill MR, Ramachandran A. Oxidant stress, mitochondria, and cell death mechanisms in drug-induced liver injury: lessons learned from acetaminophen hepatotoxicity. Drug Metab Rev. 2012;44(1):88-106.

20. Ju C, Reilly T. Role of immune reactions in drug-induced liver injury (DILI). Drug Metab Rev. 2012;44(1):107-115.

21. Njoku DB. Suppressive and pro-inflammatory roles for IL-4 in the pathogenesis of experimental drug-induced liver injury: a review. Expert Opin Drug Metab Toxicol. 2010;6(5):519-531.

22. Holt M, Ju C. Drug-induced liver injury. Handb Exp Pharmacol. 2010;196:3-27.

23. Holt MP, Ju C. Mechanisms of drug-induced liver injury. AAPS J. 2006; 8(1):E48-E54.

24. Pollak PT, Sharma AD, Carruthers SG. Relation of amiodarone hepatic and pulmonary toxicity to serum drug concentrations and superoxide dismutase activity. Am J Cardiol. 1990;65(18):1185-1191.

25. Huang YS. Genetic polymorphisms of drug-metabolizing enzymes and the susceptibility to antituberculosis drug-induced liver injury. Expert Opin Drug Metab Toxicol. 2007;3(1):1-8.

26. Behl M, Nyska A, Chhabra RS, et al. Liver toxicity and carcinogenicity in F344/N rats and B6C3F1 mice exposed to kava kava. Food Chem Toxicol. 2011;49(11):2820-2829.

27. Molleston JP, Fontana RJ, Lopez MJ, Kleiner DE, Gu J, Chalasani N. Characteristics of idiosyncratic drug-induced liver injury in children results from the DILIN prospective study. J Pediatr Gastroenterol Nutr. 2011;53(2):182-189.

28. Kaplowitz N. Drug-induced liver injury. Clin Infect Dis. 2004; 38 Suppl 2:S44-S48. 
29. Grattagliano I, Bonfrate L, Diogo CV, Wang HH, Wang DQ, Portincasa P. Biochemical mechanisms in drug-induced liver injury: certainties and doubts. World J Gastroenterol. 2009;15(39):4865-4876.

30. Ramachandran R, Kakar S. Histological patterns in drug-induced liver disease. J Clin Pathol. 2009;62(6):481-492.

31. Strazzabosco M, Fabris L, Spirli C. Pathophysiology of cholangiopathies. J Clin Gastroenterol. 2005;39(4 Suppl 2):S90-S102.

32. Orman ES, Conjeevaram HS, Vuppalanchi R, et al. Clinical and histopathologic features of fluoroquinolone-induced liver injury. Clin Gastroenterol Hepatol. 2011;9(6):517-523, e513.

33. Liu ZX, Kaplowitz N. Immune-mediated drug-induced liver disease. Clin Liver Dis. 2002;6(3):755-774.

34. Velayudham LS, Farrell GC. Drug-induced cholestasis. Expert Opin Drug Saf. 2003;2(3):287-304.

35. Derby LE, Jick H, Henry DA, Dean AD. Erythromycin-associated cholestatic hepatitis. Med J Aust. 1993;158(9):600-602.

36. Schreiber AJ, Simon FR. Estrogen-induced cholestasis: clues to pathogenesis and treatment. Hepatology. 1983;3(4):607-613.

37. Parker WA, Shearer CA. Phenytoin hepatotoxicity: a case report and review. Neurology. 1979;29(2):175-178.

38. Di Mizio G, Gambardella A, Labate A, Perna A, Ricci P, Quattrone A. Hepatonecrosis and cholangitis related to long-term phenobarbital therapy: an autopsy report of two patients. Seizure. 2007;16(7):653-656.

39. Burgunder JM, Abernethy DR, Lauterburg BH. Liver injury due to verapamil. Hepatogastroenterology. 1988;35(4):169-170.

40. Rubin E. Iatrogenic hepatic injury. Hum Pathol. 1980;11(4): 312-331.

41. Pachkoria K, Lucena MI, Molokhia M, et al. Genetic and molecular factors in drug-induced liver injury: a review. Curr Drug Saf. 2007;2(2): 97-112.

42. Andrade RJ, Robles M, Ulzurrun E, Lucena MI. Drug-induced liver injury: insights from genetic studies. Pharmacogenomics. 2009;10(9): 1467-1487.

43. Daly AK, Day CP. Genetic association studies in drug-induced liver injury. Drug Metab Rev. 2012;44(1):116-126.

44. Russmann S, Jetter A, Kullak-Ublick GA. Pharmacogenetics of druginduced liver injury. Hepatology. 2010;52(2):748-761.

45. Daly AK. Drug-induced liver injury: past, present and future. Pharmacogenomics. 2010;11(5):607-611.

46. Laverty HG, Antoine DJ, Benson C, Chaponda M, Williams D, Kevin Park B. The potential of cytokines as safety biomarkers for drug-induced liver injury. Eur J Clin Pharmacol. 2010;66(10):961-976.

47. Daly AK, Donaldson PT, Bhatnagar P, et al. HLA-B*5701 genotype is a major determinant of drug-induced liver injury due to flucloxacillin. Nat Genet. 2009;41(7):816-819.

48. Zhang M, Chen M, Tong W. Is toxicogenomics a more reliable and sensitive biomarker than conventional indicators from rats to predict drug-induced liver injury in humans? Chem Res Toxicol. 2012;25(1): $122-129$.

49. Pirmohamed M. Genetics and the potential for predictive tests in adverse drug reactions. Chem Immunol Allergy. 2012;97:18-31.

50. Zhang YN, Cui W, Han M, et al. Gene polymorphism of CYP450 2C9 and VKORC1 in Chinese population and their relationships to the maintaining dosage of warfarin. Zhonghua Liu Xing Bing Xue Za Zhi. 2010;31(2):218-222. Chinese.

51. Williams PA, Cosme J, Ward A, Angove HC, Matak Vinkovic D, Jhoti H. Crystal structure of human cytochrome P450 2C9 with bound warfarin. Nature. 2003;424(6947):464-468.

52. Higuchi N, Tahara N, Yanagihara K, et al. NAT2 6A, a haplotype of the $\mathrm{N}$-acetyltransferase 2 gene, is an important biomarker for risk of anti-tuberculosis drug-induced hepatotoxicity in Japanese patients with tuberculosis. World J Gastroenterol. 2007;13(45):6003-6008.

53. Wang K, Lee I, Carlson G, Hood L, Galas D. Systems biology and the discovery of diagnostic biomarkers. Dis Markers. 2010;28(4):199-207.

54. Soga T, Baran R, Suematsu M, et al. Differential metabolomics reveals ophthalmic acid as an oxidative stress biomarker indicating hepatic glutathione consumption. J Biol Chem. 2006;281(24):16768-16776.
55. Lee MS, Jung BH, Chung BC, et al. Metabolomics study with gas chromatography-mass spectrometry for predicting valproic acid-induced hepatotoxicity and discovery of novel biomarkers in rat urine. Int J Toxicol. 2009;28(5):392-404.

56. Young TH, Tang HS, Chao YC, et al. Quantitative rat liver function test by galactose single point method. Lab Anim. 2008;42(4):495-504.

57. Beger RD, Sun J, Schnackenberg LK. Metabolomics approaches for discovering biomarkers of drug-induced hepatotoxicity and nephrotoxicity. Toxicol Appl Pharmacol. 2010;243(2):154-166.

58. Ozer J, Ratner M, Shaw M, Bailey W, Schomaker S. The current state of serum biomarkers of hepatotoxicity. Toxicology. 2008;245(3): 194-205.

59. Nissen NI, Ranlov P, Weis-Fogh J. Evaluation of four different serum enzymes in the diagnosis of acute myocardial infarction. Br Heart $J$. $1965 ; 27: 520-526$.

60. Dufour DR, Lott JA, Nolte FS, Gretch DR, Koff RS, Seeff LB. Diagnosis and monitoring of hepatic injury. I. Performance characteristics of laboratory tests. Clin Chem. 2000;46(12):2027-2049.

61. Volkmann X, Anstaett M, Hadem J, et al. Caspase activation is associated with spontaneous recovery from acute liver failure. Hepatology. 2008;47(5):1624-1633.

62. McGill MR, Sharpe MR, Williams CD, Taha M, Curry SC, Jaeschke H. The mechanism underlying acetaminophen-induced hepatotoxicity in humans and mice involves mitochondrial damage and nuclear DNA fragmentation. J Clin Invest. 2012;122(4):1574-1583.

63. Antoine DJ, Jenkins RE, Dear JW, et al. Molecular forms of HMGB1 and keratin-18 as mechanistic biomarkers for mode of cell death and prognosis during clinical acetaminophen hepatotoxicity. $J$ Hepatol. 2012;56(5):1070-1079.

64. Rutherford A, King LY, Hynan LS, et al. Development of an accurate index for predicting outcomes of patients with acute liver failure. Gastroenterology. 2012;143(5):1237-1243.

65. Reuben A. Hy's law. Hepatology. 2004;39(2):574-578

66. Miyamoto M, Yanai M, Ookubo S, Awasaki N, Takami K, Imai R. Detection of cell-free, liver-specific mRNAs in peripheral blood from rats with hepatotoxicity: a potential toxicological biomarker for safety evaluation. Toxicol Sci. 2008;106(2):538-545.

67. Wetmore BA, Brees DJ, Singh R, et al. Quantitative analyses and transcriptomic profiling of circulating messenger RNAs as biomarkers of rat liver injury. Hepatology. 2010;51(6):2127-2139.

68. Wang K, Zhang S, Marzolf B, et al. Circulating microRNAs, potential biomarkers for drug-induced liver injury. Proc Natl Acad Sci U S A. 2009;106(11):4402-4407.

69. Yokoi T, Nakajima M. Toxicological implications of modulation of gene expression by microRNAs. Toxicol Sci. 2011;123(1):1-14.

70. Starkey Lewis PJ, Dear J, Platt V, et al. Circulating microRNAs as potential markers of human drug-induced liver injury. Hepatology. 2011;54(5):1767-1776.

71. Zhang Y, Jia Y, Zheng R, et al. Plasma microRNA-122 as a biomarker for viral-, alcohol-, and chemical-related hepatic diseases. Clin Chem. 2010;56(12):1830-1838.

72. Stine JG, Lewis JH. Drug-induced liver injury: a summary of recent advances. Expert Opin Drug Metab Toxicol. 2011;7(7):875-890.

73. Perez Alea M, Kitamura M, Martin G, Thomas V, Hitomi K, El Alaoui S. Development of an isoenzyme-specific colorimetric assay for tissue transglutaminase 2 cross-linking activity. Anal Biochem. 2009;389(2): $150-156$.

74. James LP, Letzig L, Simpson PM, et al. Pharmacokinetics of acetaminophen-protein adducts in adults with acetaminophen overdose and acute liver failure. Drug Metab Dispos. 2009;37(8):1779-1784.

75. Qin S, Zhou Y, Lok AS, et al. SRM targeted proteomics in search for biomarkers of HCV-induced progression of fibrosis to cirrhosis in HALT-C patients. Proteomics. 2012;12(8):1244-1252.

76. Chladek J, Simkova M, Vaneckova J, Hroch M, Vavrova J, Hulek P. Assessment of methotrexate hepatotoxicity in psoriasis patients: a prospective evaluation of four serum fibrosis markers. $J$ Eur Acad Dermatol Venereol. August 7, 2012. [Epub ahead of print.] 
77. Chen M, Vijay V, Shi Q, Liu Z, Fang H, Tong W. FDA-approved drug labeling for the study of drug-induced liver injury. Drug Discov Today. 2011;16(15-16):697-703.

78. Surinova S, Schiess R, Huttenhain R, Cerciello F, Wollscheid B, Aebersold R. On the development of plasma protein biomarkers. J Proteome Res. 2011;10(1):5-16.

79. Evans C, Noirel J, Ow SY, et al. An insight into iTRAQ: where do we stand now? Anal Bioanal Chem. 2012;404(4):1011-1027.

80. Cappadona S, Baker PR, Cutillas PR, Heck AJ, van Breukelen B. Current challenges in software solutions for mass spectrometry-based quantitative proteomics. Amino Acids. 2012;43(3):1087-1108.

81. Gillet LC, Navarro P, Tate S, et al. Targeted data extraction of the MS/ MS spectra generated by data-independent acquisition: a new concept for consistent and accurate proteome analysis. Mol Cell Proteomics. 2012;11(6):O111.016717.

82. Berglund L, Bjorling E, Oksvold P, et al. A genecentric Human Protein Atlas for expression profiles based on antibodies. Mol Cell Proteomics. 2008;7(10):2019-2027.

83. Shi T, Su D, Liu T, et al. Advancing the sensitivity of selected reaction monitoring-based targeted quantitative proteomics. Proteomics. 2012;12(8):1074-1092.

84. Picotti P, Aebersold R. Selected reaction monitoring-based proteomics: workflows, potential, pitfalls and future directions. Nat Methods. 2012;9(6):555-566.
85. Woodhead JL, Howell BA, Yang Y, et al. An analysis of N-acetylcysteine treatment for acetaminophen overdose using a systems model of druginduced liver injury. J Pharmacol Exp Ther. 2012;342(2):529-540.

86. Anderson NL, Anderson NG. The human plasma proteome: history, character, and diagnostic prospects. Mol Cell Proteomics. 2002;1(11):845-867.

87. Apweiler R, Aslanidis C, Deufel T, et al. Approaching clinical proteomics: current state and future fields of application in fluid proteomics. Clin Chem Lab Med. 2009;47(6):724-744.

88. Griffiths WJ, Koal T, Wang Y, Kohl M, Enot DP, Deigner HP. Targeted metabolomics for biomarker discovery. Angew Chem Int Ed Engl. 2010;49(32):5426-5445.

89. Wishart DS, Tzur D, Knox C, et al. HMDB: the Human Metabolome Database. Nucleic Acids Res. 2007;35(Database issue):D521-D526.

90. Rhee EP, Gerszten RE. Metabolomics and cardiovascular biomarker discovery. Clin Chem. 2012;58(1):139-147.

91. Cho YE, Singh TS, Lee HC, et al. In-depth identification of pathways related to cisplatin-induced hepatotoxicity through an integrative method based on an informatics-assisted label-free protein quantitation and microarray gene expression approach. Mol Cell Proteomics. 2012;11(1):M111.010884.

92. Waters MD, Fostel JM. Toxicogenomics and systems toxicology: aims and prospects. Nat Rev Genet. 2004;5(12):936-948.
Current Biomarker Findings

\section{Publish your work in this journal}

Current Biomarker Findings is an international, peer-reviewed, open access journal publishing original research, reports, reviews and commentaries on all areas of biomarker research. The manuscript management system is completely online and includes a very quick and fair

\section{Dovepress}

peer-review system. Visit http://www.dovepress.com/testimonials.php to read real quotes from published authors. 